\title{
Residual solvent content in conducting polymer-blend films mapped with scanning transmission $x$-ray microscopy
}

\author{
Robert Meier, Markus Schindler, and Peter Müller-Buschbaum \\ Physik Department, Lehrstuhl für Funktionelle Materialien, Technische Universität München, \\ James-Franck-Strasse 1, DE-85747 Garching, Germany \\ Benjamin Watts \\ Paul Scherrer Institut, CH-5232 Villigen PSI, Switzerland \\ (Received 7 October 2011; published 10 November 2011)
}

\begin{abstract}
Near-edge x-ray absorption fine-structure spectra prove the presence of solvent molecules in conducting polymer films and are used to calculate the absolute solvent uptake of, e.g., 5 vol.\% in poly(vinylcarbazole) (PVK) films, which were prepared by solution casting with cyclohexanone as solvent. Nanoscale scanning transmission $\mathrm{x}$-ray microscopy (STXM) reveals a thickness-independent solvent content in a PVK gradient sample due to the formation of an enrichment layer of residual solvent. In polymer-blend films of PVK and poly(3-hexylthiophene) (P3HT), STXM probes a lateral residual solvent uptake, which depends on the composition of the phase-separation domains. For all measurements, oxygen-containing solvent molecules in oxygen-free conducting polymer films are used as marker material, and a significant amount of residual solvent is found in all types of investigated samples.
\end{abstract}

DOI: 10.1103/PhysRevB.84.174205

PACS number(s): 78.70.Dm, 68.37.Yz, 78.66.Qn, 81.05.Fb

\section{INTRODUCTION}

Conducting polymers have been in focus of worldwide research in recent years due to an expectation of an impending and long-term demand for polymer-based electronic devices, which can be mass produced at much lower costs than competing technologies. ${ }^{1}$ The cost benefits in fabrication result from the ability of conducting polymers to be easily processed via various simple solution-based casting techniques such as spin coating, doctor blading, and spray coating. Furthermore, organic electronic devices can be processed in ambient pressure conditions and, thus, do not need the expensive high-vacuum steps required by their inorganic semiconductor competitors. Often, the choice of solvent and post-casting annealing steps are crucial for the final device performance since they can drastically influence the resulting film morphology. ${ }^{2-5}$ For example, blend films based on the polyfluorene copolymers F8BT and PFB exhibit a strong lateral phase-separation structure (on the order of microns) if cast from xylene solution. On the other hand, such blend films cast from chloroform reveal a phase separation on the nanoscale. ${ }^{6,7}$ Up to now, one almost completely neglected area of conducting polymer research involves the presence and effects of residual solvent. Recently, Chang et al. have shown that in polymer:fullerene bulk-heterojunction solar cells, residual solvent is retained in the film leading to an extensive phase separation. ${ }^{8}$ Based on results from different thermally annealed samples, they infer that a larger amount of excess solvent remaining in the film results in higher diffusion rates of the fullerene, which strongly affects the overall power conversion efficiency. Hence, such additional solvent molecules can, over time, alter the film morphology and crystallinity of both homopolymer and blend films due to an energetic shifting of the free enthalpy (softening effect). Moreover, a strong chemical influence on the polymer chains is probable, which can result in complex aging effects, as will be discussed below.
For nonconducting polymer films, the solvent retention, according to the free-volume theory, has already been determined via different methods, unfortunately all lacking lateral resolution or absolute indication of quantity. By redissolving the already-cast polymer film in a different solvent, gas chromatography has been used to determine the residual solvent content in polystyrene films. ${ }^{9}$ Neutron reflectivity has also shown similar results for deuterated solvents (solvent uptake between 8 and 19 vol.\%). ${ }^{10}$ In addition, laterally unresolved dielectric spectroscopy has proven the presence of solvent molecules in conventional poly(methylmethacrylate) films. ${ }^{11}$ However, this question needs to also be addressed for conducting polymers, for which the effects of residual solvent could be more important in practical applications due to the extended range of material properties being utilized. From a molecular point of view, it is known that, due to the rigid backbone of the conducting polymers in comparison to the more flexible nonconducting ones, more static free volume due to less dense packing is present, which hence might even favor the retention of solvent. ${ }^{12}$

Here, we report first near-edge x-ray absorption finestructure (NEXAFS) spectroscopy and scanning transmission $\mathrm{x}$-ray microscopy (STXM) measurements on conducting polymer films probing the residual solvent content in homopolymer and segregated polymer blend films. Therefore, retained solvent molecules that contain oxygen atoms are of special interest here because photoactive materials such as oxygen are known to decrease the electroluminescence of conducting polymer-based light-emitting diodes. ${ }^{13}$ Further, the photooxidation observed in other conducting polymer films indicates a degradation of the chemical structure due to a possible chain scission or a ring opening. ${ }^{14}$ Although oxygenic solvent molecules might not be as reactive as molecular oxygen, they can be strongly polar as in the case of cyclohexanone and, therefore, also lead to chemical modifications of the polymer. Hence, the presence of retained oxygenic solvent molecules in 
an active polymer layer could be significant for the efficiency and lifetime of electronic devices, even if the device is thermally annealed and encapsulated from the surrounding environment. In general, it has been shown for nonconducting ${ }^{9-11}$ and for conducting polymers ${ }^{8}$ that the solvent removal in the films due to thermal annealing is rather complex and often not all solvent residuals are removed. For investigations of different polymer-blend systems, STXM and NEXAFS measurements have already proven their suitability due to a high spatial resolution in combination with strong chemical contrast. ${ }^{15-17}$ Burke et $a l .{ }^{18}$ have used STXM to indirectly prove solvent trapping in ternary blend films dedicated to organic solar cells. These films showed submicron depressions upon annealing, which were caused by the evaporation of the embedded solvent and depend strongly on the film composition.

\section{EXPERIMENTAL METHODS}

\section{A. Sample preparation}

P3HT was supplied from Rieke Metals, Inc. (Lincoln, USA) and had a molecular weight of $50 \times 10^{3} \mathrm{~g} / \mathrm{mol}$. PVK was ordered from Sigma Aldrich (Seelze, Germany) at a molecular weight of $1.1 \times 10^{6} \mathrm{~g} / \mathrm{mol}$. Both polymers are widely used and their characteristics can be found elsewhere. ${ }^{19,20}$ The materials were used as supplied. For the PVK homopolymer films, the polymer was dissolved in cyclohexanone at a concentration of $40 \mathrm{~g} / \mathrm{l}$. The solution was directly cast on nitride membranes, which were purchased from Silson (Blisworth, England). The PVK homopolymer samples were prepared in a glove box and the samples were handled and stored under a nitrogen atmosphere. The PVK thickness gradient sample was fabricated via solution casting of a $40 \mathrm{~g} / \mathrm{l} \mathrm{PVK}$ in cyclohexanone solution onto an inclined substrate (inclination angle $25^{\circ}$ ). The casting was performed directly on the $\mathrm{SiN}$-membrane support with the solution retained by a sharp silicon dam.

For the P3HT:PVK blend films, both components were dissolved separately in tetrahydrofuran (THF) before mixing at a volume ratio of 80:20. For the PVK solution, a concentration of $10 \mathrm{~g} / \mathrm{l}$ was chosen and, for the P3HT, $8 \mathrm{~g} / \mathrm{l}$. The polymer blend solution was then spin coated on an acidic precleaned silicon substrate. ${ }^{21}$ The spin coating was performed on a Süss MicroTec Delta6RC spin coater under ambient conditions at $2000 \mathrm{rpm}$ for $60 \mathrm{~s}$. The polymer blend film is then transferred via floating to a copper TEM grid, which was purchased from Plano (Wetzlar, Germany).

\section{B. Near-edge $x$-ray absorption fine-structure spectra}

The NEXAFS measurements were performed at the PolLux beamline at the Swiss light Source (Villigen, Switzerland), which offers an extended photon energy range suited for measurements at the carbon and oxygen $K$-edge (200$1400 \mathrm{eV}){ }^{22}$ In order to allow for improved statistics, the NEXAFS spectra were recorded with an unfocused x-ray beam by taking out the focusing Fresnel zone plate. For the calculation of the solvent content (see Fig. 1), the spectra were recorded between 510 and $590 \mathrm{eV}$ at a step size of $0.5 \mathrm{eV}$ and each data point was integrated over $50 \mathrm{~ms}$. For the calculation of the polymer-blend compositional map [see Fig. 3(b)], the spectra were taken at the carbon edge between 280 and

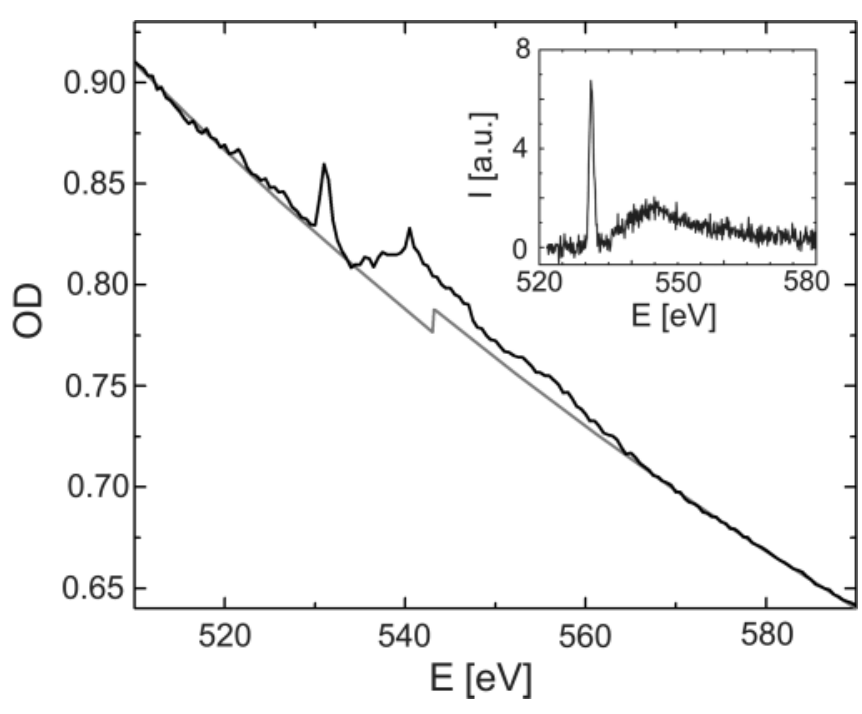

FIG. 1. NEXAFS spectrum of a PVK film prepared from cyclohexanone solution probed at the oxygen $K$-edge (black line). The gray line is calculated from a theoretical model based on the chemical composition of the film and can be used to determine the solvent content in thin films. The inset shows the corresponding EELS converted to an oscillator strength per oxygen atom of pure cyclohexanone (Refs. 28 and 29).

$320 \mathrm{eV}$ with the experimental conditions from above. In order to prevent absorption from the surrounding atmosphere, the measurements were performed at a chamber pressure of $9.0 \times 10^{-3}$ mbar. Additional information about NEXAFS and the corresponding data analysis can be found in literature. ${ }^{23}$

\section{Scanning transmission $x-$ ray microscopy}

Also, the STXM data were taken at the PolLux beamline, which allows a lateral resolution of down to $15 \mathrm{~nm}^{24}$ Therefore, the sample is rastered with respect to the $\mathrm{x}$-ray beam and the transmission signal is detected by a photomultiplier tube. All STXM images were recorded with a resolution of $100 \times 100$ data points with an integrating time of $200 \mathrm{~ms}$ per pixel. The compositional map of Fig. 3(b) was derived using the IDL widget aXis 2000 (Ref. 25) by the following process. The lateral drifting between images at different energies was corrected by automatically aligning the images in respect to each other. The NEXAFS spectra of the pristine P3HT and PVK were normalized by the film thickness and a sum of them is fitted to the measured blend spectra. This analysis is performed at each data point, and the resulting coefficients were used to calculate the film composition. Additional information can be found elsewhere. ${ }^{17,18,26}$ A similar data analysis was also performed for Fig. 4.

\section{Atomic-force microscopy}

The surface topography was imaged with atomic-force microscopy in tapping-mode condition [see Fig. 3(a)]. The measurement was carried out with an Autoprobe CP Research AFM instrument and it was performed in ambient air. Gold-coated silicon cantilevers (Ultralever cantilevers) with a resonance frequency of $75 \mathrm{kHz}$ and a spring constant of 
2.1 $\mathrm{Nm}^{-1}$ were used. The curvature of the tip was small compared to the measured structure size.

\section{RESULTS AND DISCUSSION}

\section{A. Solvent content in conducting homopolymer films}

In this work, we explicitly investigate the retention of oxygen-containing solvent molecules in oxygen-free polymer films using a combination of NEXAFS spectroscopy (C $K$-edge and $\mathrm{O} K$-edge) and STXM. The fact that oxygen atoms are found only in the solvent makes it an ideal marker element for $\mathrm{x}$-ray spectroscopy, while the unique $\mathrm{C} K$-edge spectra of the polymer materials provide another contrast mechanism that allows us to identify and quantify each of the components in our sample films. While these techniques are not new to the field of STXM, these measurements are the first to be applied to a polymer:retained solvent system.

Homopolymer films are made of the oxygen-free poly(vinylcarbazole) (PVK), which is an important component for the fabrication of white-light-emitting diodes with high luminous efficiencies. ${ }^{27}$ Thus, by combination of oxygenfree conducting polymers and oxygen-containing solvents, in our case cyclohexanone, the necessary contrast is gained to examine complex polymer:retained solvent systems with soft $\mathrm{x}$-rays. Figure 1 shows the $\mathrm{O} K$-edge NEXAFS spectrum of a $600 \mathrm{~nm}$ thick homogeneous PVK film cast from a $40 \mathrm{~g} / 1$ cyclohexanone solution (black line). The absorption spectrum contains spectral features identical to the electron energy loss spectrum (EELS) of pure cyclohexanone shown in the inset. $^{28,29}$ In both cases, a prominent absorption maximum at $531.5 \mathrm{eV}$ (attributed to $\mathrm{O} 1 s \rightarrow \pi_{C=O}^{*}$ transitions) is accompanied by a broader and less intense set of absorption peaks between 530 and $560 \mathrm{eV}$ (attributed to $\mathrm{O} 1 s \rightarrow \sigma_{C=O}^{*}$ transitions). This spectrum therefore testifies that the observed oxygen signal is indeed due to retained solvent molecules and not to the presence of water, air, or oxidation of the polymer or substrate. Moreover, the spectrum of the substrate was eliminated by the practice of using measurements on a blank nitride membrane for beam intensity corrections. The NEXAFS spectrum also shows an additional steplike increase in absorption that corresponds to transitions to the continuum and indicates the overall oxygen content. A theoretical model for the X-ray absorption of the materials was constructed from the chemical composition of PVK $\left(\mathrm{C}_{14} \mathrm{H}_{11} \mathrm{~N}\right)$ and cyclohexanone $\left(\mathrm{C}_{6} \mathrm{H}_{10} \mathrm{O}\right)$ and the atomic scattering factors published by Henke et al. ${ }^{30}$ (shown as a gray line in Fig. 1). Fitting this model to the data yielded the percentage solvent retained within the polymer film. Since the Henke atomic scattering factors do not include near-edge resonance effects, the fit of the model to the data must be evaluated only by the absorption step-edge component of the measured spectrum. Thus, the sum of all absorption coefficients of polymer and solvent at some ratio are simulated in order to fit the measured optical density (OD) in the pre- and post-edge regions:

$$
\mathrm{OD}=-\ln \frac{I}{I_{0}}=\rho \mu_{\mathrm{tot}} d
$$

with

$$
\mu_{\mathrm{tot}}=\alpha \mu_{\mathrm{pol}}+\beta \mu_{\mathrm{sol}}=\alpha \sum_{p} n_{q, p} \mu_{a, p}+\beta \sum_{s} n_{q, s} \mu_{a, s} .
$$

According to the Lambert-Beer law, $\rho$ is the concentration of absorbing species, $\mu$ the various absorption coefficients, and $d$ the film thickness. The percentaged polymer and solvent content is described by $\alpha$ and $\beta$, respectively, and the atomic absorption coefficients of the present atoms by $\mu_{p}$ and $\mu_{s}$ depending on their corresponding relative weights $n_{q, p}$ and $n_{q, s}$. By taking into account the mass density of polymer and solvent, an overall volume content of the cyclohexanone in the PVK film of $5 \%$ is found. To the best of our knowledge, this is the first measured value for the solvent retention in solutioncast thin conducting polymer films. In comparison, standard coiled PS films prepared from toluene solution revealed an amount of residual solvent between 8 and 19 vol.\% depending on molecular weight and film thickness. ${ }^{9,10}$

\section{B. Dependence of solvent content on polymer-film thickness}

In order to investigate the relationship between film thickness and residual solvent content in conducting PVK films, the lateral resolution of the STXM setup allows the mapping of a thickness gradient sample. Figure 2(a) shows the transmitted intensity taken at the photon energy of $520 \mathrm{eV}$, which is in the oxygen $K$-pre-edge region and, hence, can be used as a logarithmic measure of the film thickness according to the Lambert-Beer law. By an additional intensity map of the same sample area, utilizing the sharp absorption peak of the cyclohexanone at $531.5 \mathrm{eV}$, the spatially resolved optical

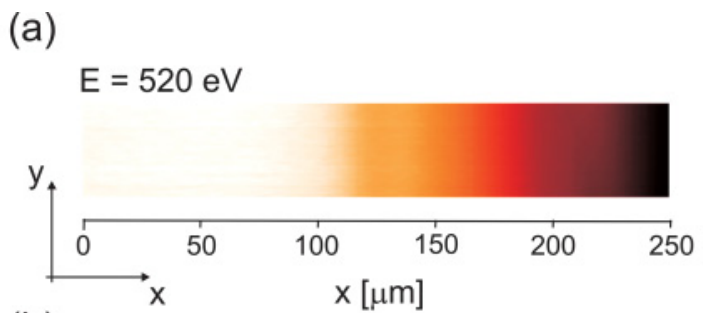

(b)

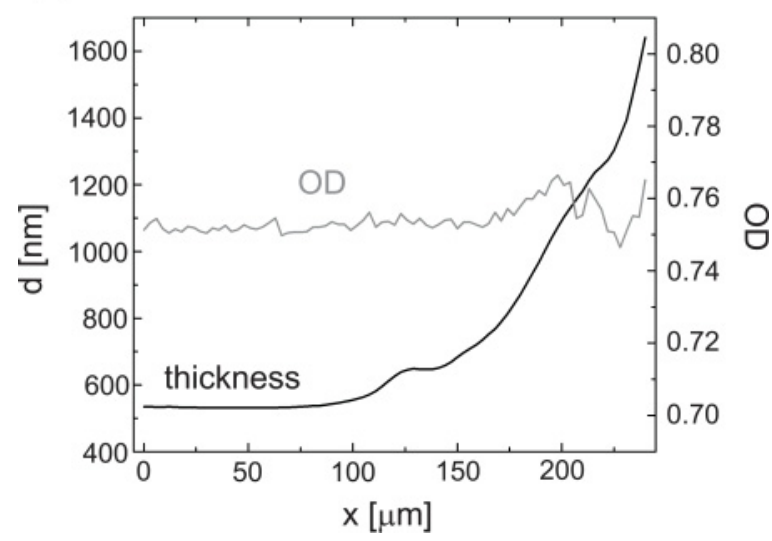

FIG. 2. (Color online) (a) Two-dimensional STXM image of a solution-cast PVK thickness gradient recorded at $520 \mathrm{eV}$. The decreasing transmitted intensity depicts the increasing film thickness. (b) Optical density (OD) of oxygen and film thickness $d$ plotted as function of the lateral $x$ position. 
density of this oxygen $\pi^{*}$ resonance is calculated. Since the film thickness gradient is only present along the $x$ axis, whereas the thickness is homogeneous in the $y$ direction, an integrated line profile is generated to gain improved statistics. Figure 2(b) compares the variation of film thickness with $x$ position to the corresponding calculated optical density of oxygen. In contrast to the monotonically increasing PVK thickness (increasing from 530 to $1630 \mathrm{~nm}$ ), the measured oxygen optical density remains constant at $\approx 0.76$. This behavior indicates that the retained solvent likely exists at or near an interface, rather than within the bulk of the polymer film. In addition, the measurement of the film thickness gradient further verifies that oxygen present in the film can only be attributed to the solvent, as it was already indicated by the spectral absorption features in the NEXAFS spectrum in Fig. 1. A prior oxidization of the conducting polymer, which could occur between polymer synthesis and coating, would result in a strong thickness dependence.

\section{Laterally distributed retained solvent content in polymer blend films}

The high lateral resolution of STXM is especially useful for imaging the morphology of polymer blend films, which often show nonequilibrium phase-separation structures on the nanoscale and microscale. ${ }^{31}$ For nonequilibrium phaseseparation structures of polymer blend films, STXM not only reveals the lateral dimensions of the segregated polymer domains, but also their composition by employing the corresponding NEXAFS spectra at the carbon $K$-edge. By focusing on an oxygen-free polymer blend system, spin coated from a common oxygenic solvent, STXM images allow the calculation of two-dimensional residual solvent maps. Therefore, the already discussed PVK is blended with poly(3-hexylthiophene) (P3HT) in THF, and thin films were prepared via spin coating. The electroluminescence from such polymer blend thin films has been intensely studied for application in light-emitting diodes (LED). ${ }^{32}$ Currently, also a P3HT- $b$-PVK diblock copolymer has been used to fabricate white-light-emitting diodes. ${ }^{33}$ The atomic-force-microscopy image (AFM) in Fig. 3(a) shows the topography of the polymer blend film with a maximum peak-to-valley distance of $101 \mathrm{~nm}$
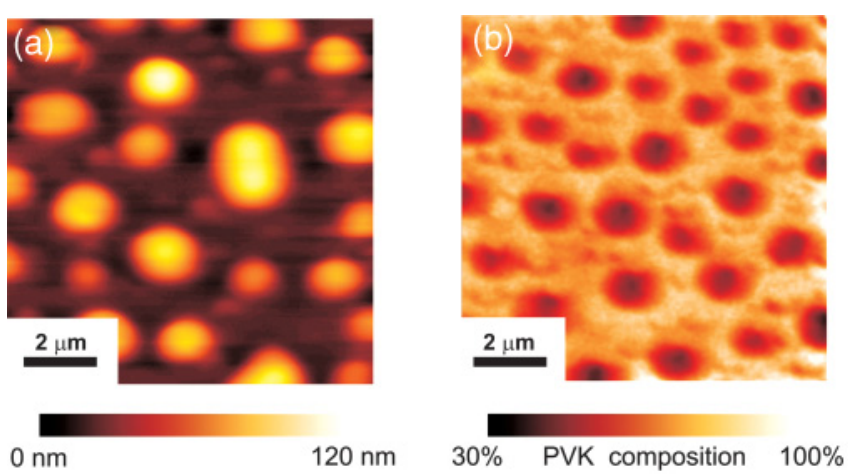

FIG. 3. (Color online) (a) AFM image of surface topology of P3HT:PVK polymer-blend film with a peak-to-valley distance of $101 \mathrm{~nm}$. (b) Quantitative PVK compositional map calculated from STXM images taken at different photon energies of 280, 284.5, and $287.5 \mathrm{eV}$ at a mean film thickness of $160 \mathrm{~nm}$. The circular polymer domains have a diameter of $\approx 0.5-1.6 \mu \mathrm{m}$ and are randomly distributed. The composition map, shown in Fig. 3(b), is calculated from multiple STXM images at energies located at prominent spectral features from the NEXAFS spectra at the carbon $K$-edge in combination with the spectra of the pristine components. ${ }^{34,35}$ Figure 3 shows that the elevated phase has $\mathrm{P} 3 \mathrm{HT}$ as the majority component $(\approx 70 \%)$, whereas the surrounding polymer matrix is almost entirely composed of PVK. Both methods show a smooth transition between the polymer domains with respect to film thickness and composition. Such smooth transition is different to other conducting polymer blend films such as F8BT:PFB systems, which exhibited distinct polymer domain transitions as probed with similar methods by McNeill et al.$^{35,36}$ Complementary STXM images with higher resolution were made, which show no smaller structures than observed in Fig. 3(b) to the limit of $35 \mathrm{~nm}$ resolution. In addition, the exceptional highly crystalline character of $\mathrm{P} 3 \mathrm{HT}$ leads to a more ordered polymerchain arrangement on a molecular level in comparison with PVK. This could result in a preference for solvent to be retained in particular domains of a polymer blend film and, hence, an uneven spatial distribution. In order to address the idea of an uneven spatial solvent distribution in such blend films, STXM images were again taken at photon energies corresponding to the oxygen $K$-pre-edge region [525 eV, Fig. 4(a)] and a prominent spectral feature from THF $[531.5 \mathrm{eV}$, Fig. 4(b)]. While each of these images depends strongly on the variations in film thickness, this dependence cancels
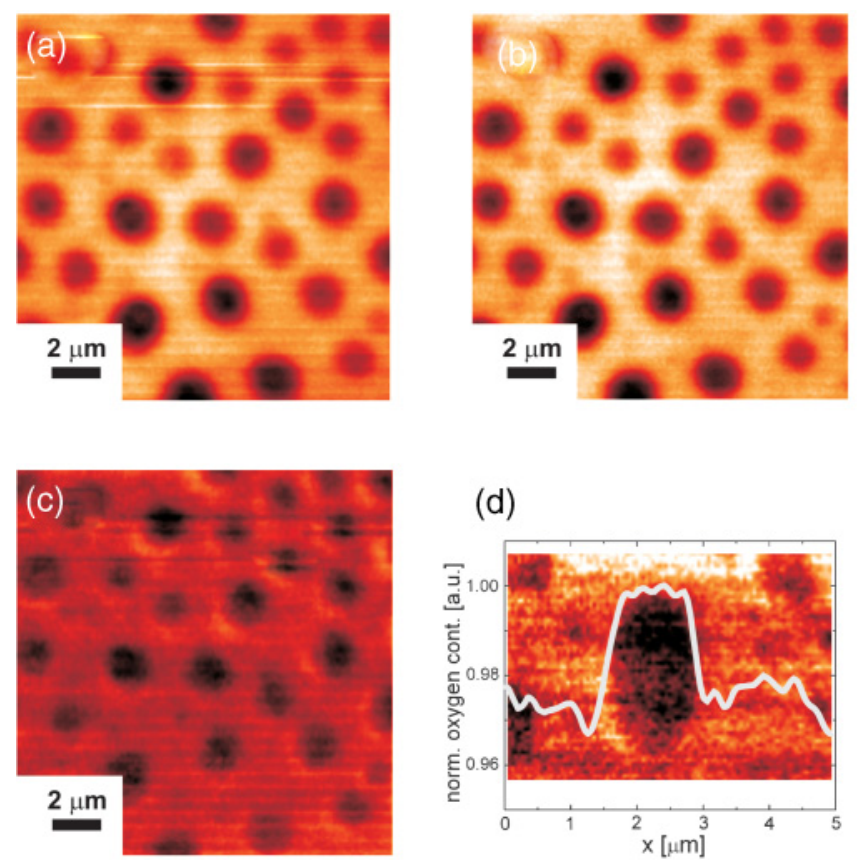

(d)

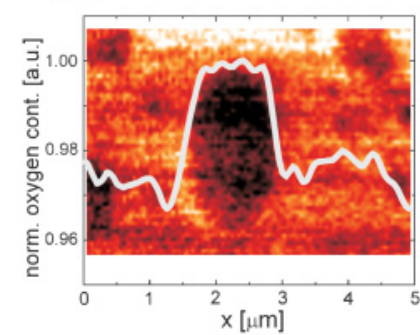

FIG. 4. (Color online) STXM microscopy images taken at (a) $525 \mathrm{eV}$ and (b) $531.5 \mathrm{eV}$. According to these images, (c) represents the solvent distribution in the polymer blend, which matches the phase-separation structure of the polymer blend. (d) Close-up: A line cut of the absorbance along the P3HT-enriched polymer domain reveals a higher solvent content in comparison to the surrounding PVK matrix. 
out in their ratio, leaving a map that displays the spatial distribution of THF [Fig. 4(c)]. Because it was formed from raw transmission images, the contrast of the THF map is such that greater amounts of THF appear darker. Interestingly, Fig. 4(c) shows an identical pattern to that of the film's phase-separation morphology seen in the separate STXM and AFM images presented in Fig. 3. A greater amount of residual THF solvent is seen in the P3HT-rich domains as compared to the PVK-rich matrix. Figure 4(d) shows a line cut across a P3HT-rich polymer domain plotted over the higher-resolution STXM image it was extracted from. For convenience, the intensity of the pristine line cut is inverted to represent the solvent content and normalized to the maximum value of the oxygen found in the P3HT domains. The normalized peak-to-valley ratio of oxygen of $\approx 3 \%$ suggests overall small deviations from the mean solvent distribution. Nevertheless, the strong increase in the line cut at the polymer phase boundaries shows a well-defined preferred residue of solvent molecules in the P3HT-rich domains. For the determination of absolute solvent content values in the two polymer-blend domains, similar NEXAFS spectra as shown in Fig. 1 would be necessary. Unfortunately, such measurements are only possible using an unfocused setup due to the low amount of solvent present.

From a chemical point of view, it is known that $\mathrm{H}$ atoms as present in THF can interact with the thienyl backbone bearing $\pi$ system. ${ }^{37}$ In principle, similar weak $\pi-\mathrm{H}$ interactions are also possible for the carbazole units in the PVK. Nevertheless, it can be assumed that strong $\pi-\pi$ interactions lead to a denser packing of the more flexible PVK chains in comparison to the rigid $\mathrm{P} 3 \mathrm{HT}$, hindered also by its side chains. Based on steric restraints, this leads to a reduced effective surface for the solvent molecules to attach to a polymer chain in the PVKrich phase. Due to an increased free volume in the crystalline entanglement of the P3HT-rich domains, the solvent molecules could also be preferentially trapped in such voids.

\section{CONCLUSIONS}

In summary, we have introduced a field of application for NEXAFS and two-dimensional STXM measurements. The presence of residual solvent molecules embedded in thin conducting polymer films prepared via solution casting is proven by absorption spectra, and the absolute amount of residual solvent is calculated by simulating the oxygen $K$-absorption edge with an absorption-coefficient-based model. STXM images of a thickness gradient sample show a thickness-independent amount of residual solvent, indicating the presence of a solvent enrichment layer. In polymer blends, the amount of residual solvent depends on the interaction of the polymer and thus varies locally with the phase-separation structure. However, residual solvent is found in all types of investigated samples, which is a significant result due to the potential influence on the performance and longevity of the polymer electronic devices currently under development.

\section{ACKNOWLEDGMENTS}

The financial support by the BMBF Project No. 05KS7W01 is gratefully acknowledged. PolLux is funded by the BMBF (Project No. 05KS7WE1).
${ }^{1}$ C. Lungenschmied, G. Dennler, H. Neugebauer, N. S. Sariciftci, M. Glatthaar, T. Meyer, and A. Meyer, Sol. Energy Mater. Sol. Cells. 91, 379 (2007).

${ }^{2}$ H. Hoppe and N. S. Sariciftci, J. Mater. Chem. 16, 45 (2005).

${ }^{3}$ M. A. Ruderer, S. Guo, R. Meier, H.-Y. Chiang, V. Körstgens, J. Wiedersich, J. Perlich, S. V. Roth, and P. Müller-Buschbaum, Adv. Funct. Mater. 21, 3382 (2011).

${ }^{4}$ J. W. Kiel, A. P. R. Eberle, and M. E. Mackay, Phys. Rev. Lett. 105, 168701 (2010).

${ }^{5}$ T. Kirchartz, J. Mattheis, and U. Rau, Phys. Rev. B 78, 235320 (2008).

${ }^{6}$ A. C. Arias, J. D. MacKenzie, R. Stevenson, J. J. M. Halls, M. Inbasekaran, E. P. Woo, D. Richards, and R. H. Friend, Macromolecules (Washington DC, US) 34, 6005 (2001).

${ }^{7}$ S. Westenhoff, I. A. Howard, and R. H. Friend, Phys. Rev. Lett. 101, 016102 (2008).

${ }^{8}$ L. Chang, H. W. A. Lademann, J.-B. Bonekamp, K. Meerholz, and A. J. Moule, Adv. Funct. Mater. 21, 1779 (2011).

${ }^{9}$ J. Garcia-Turiel and B. Jerome, Colloid Polym. Sci. 285, 1617 (2007).

${ }^{10}$ J. Perlich, V. Körstgens, E. Metwalli, L. Schulz, R. Georgii, and P. Müller-Buschbaum, Macromolecules (Washington DC, US) 42, 337 (2009).
${ }^{11}$ S. Bistac and J. Schultz, Progr. Org. Coating 31, 347 (1997).

${ }^{12}$ K. Choi and W. H. Jo, Macromolecules (Washington DC, US) 28, 8598 (1995).

${ }^{13}$ Y. Kaminorz, E. Smela, O. Inganäs, and L. Brehmer, Adv. Mater. 10, 765 (1998).

${ }^{14} \mathrm{~N}$. Ljungqvist and T. Hjertberg, Macromolecules (Washington DC, US) 28, 5993 (1995).

${ }^{15}$ H. Ade and H. Stoll, Nat. Mater. 8, 281 (2009).

${ }^{16}$ M. D. de Jonge, B. Hornberger, C. Holzner, D. Legnini, D. Paterson, I. McNulty, C. Jacobsen, and S. Vogt, Phys. Rev. Lett. 100, 163902 (2008).

${ }^{17}$ C. R. McNeill, B. Watts, L. Thomsen, W. J. Belcher, N. C. Greenham, and P. C. Dastoor, Nano Lett. 6, 1202 (2006).

${ }^{18}$ K. B. Burke, W. J. Belcher, B. Watts, C. R. McNeill, H. Ade, and P. C. Dastoor, Macromolecules (Washington DC, US) 42, 3098 (2009).

${ }^{19}$ G. Kaune, W. Wang, E. Metwalli, M. Ruderer, R. Roßner, S. Roth, and P. Müller-Buschbaum, Eur. Phys. J. E 26, 73 (2008).

${ }^{20}$ C. Deibel, D. Mack, J. Gorenflot, A. Schöll, S. Krause, F. Reinert, D. Rauh, and V. Dyakonov, Phys. Rev. B 81, 085202 (2010).

${ }^{21}$ P. Müller-Buschbaum, Eur. Phys. J. E 12, 443 (2003). 
${ }^{22}$ J. Raabe, G. Tzvetkov, U. Flechsig, M. Böge, A. Jaggi, B. Sarafimov, M. G. C. Vernooij, T. Huthwelker, H. Ade, D. Kicoyne, T. Tyliszczak, R. H. Fink, and C. Quitmann, Rev. Sci. Instrum. 79, 113704 (2008).

${ }^{23}$ B. Watts, L. Thomsen, and P. C. Dastoor, J. Electron Spectrosc. Relat. Phenom. 151, 105 (2006).

${ }^{24}$ K. Jefimovs, J. Vila-Comamala, T. Pilvi, J. Raabe, M. Ritala, and C. David, Phys. Rev. Lett. 99, 264801 (2007).

${ }^{25}$ A. Hitchcock [http://unicorn.mcmaster.ca/aXis2000.html].

${ }^{26}$ B. Watts and C. R. McNeill, Macromol. Rapid Commun. 31, 1706 (2010).

${ }^{27}$ J. Zou, J. Liu, H. Wu, W. Yang, and J. P. Y. Cao, Org. Electr. 10, 843 (2009).

${ }^{28}$ J. T. Francis and A. P. Hitchcock, J. Phys. Chem. 98, 3650 (1994).

${ }^{29}$ A. P. Hitchcock [http://unicorn.mcmaster.ca/corex/cedb-title.html].

${ }^{30}$ B. L. Henke, E. Gullikson, and J. Davis, At. Data Nucl. Data Tables 54, 181 (1993).
${ }^{31}$ R. Meier, M. A. Ruderer, A. Diethert, G. Kaune, V. Körstgens, S. V. Roth, and P. Müller-Buschbaum, J. Phys. Chem. B 115, 2899 (2011).

${ }^{32}$ H. Nishino, G. Yu, A. J. Heeger, T.-A. Chen, and R. D. Rieke, Synth. Met. 68, 243 (1995).

${ }^{33}$ M. Heo, J. Kim, J. Y. Kim, and C. Yang, Macromol. Rapid Commun. 31, 2047 (2010).

${ }^{34}$ H. Ade, X. Zhang, S. Cameron, C. Costello, J. Kirz, and S. Williams, Science 258, 972 (1992).

${ }^{35}$ C. R. McNeill, B. Watts, L. Thomsen, H. Ade, N. C. Greenham, and P. C. Dastoor, Macromolecules (Washington DC, US) 40, 3263 (2007).

${ }^{36}$ M. A. Ruderer and P. Müller-Buschbaum, Soft Matter 7, 5482 (2011).

${ }^{37}$ D. H. Kim, Y. D. Park, Y. Jang, H. Yang, Y. H. Kim, J. I. Han, D. G. Moon, S. Park, T. Chang, C. Chang, M. Joo, C. Y. Ryu, and K. Cho, Adv. Funct. Mater. 15, 77 (2005). 